Cell Research (1999), 9, 281-290

\title{
Nuclear pseudogenes of mitochondrial DNA as a variable part of the human genome
}

\author{
Yuan Jin Duo ${ }^{1,2}$, Jin Xiu SHI ${ }^{1}$, Guang Xun \\ $\mathrm{MENG}^{1,2}$, Li Guo AN ${ }^{2}$, Geng Xi HU ${ }^{1}$ \\ 1. Shanghai Institute of Cell Biology and the Shanghai Life \\ Science Center, Chinese Academy of Sciences, 320 Yue Yang \\ Road, Shanghai 200031, China \\ 2. Department of Biology, Shandong Normal University, Jinan \\ 250014, China
}

\begin{abstract}
Novel pseudogenes homologous to the mitochondrial (mt) 16S rRNA gene were detected via different approaches. Eight pseudogenes were sequenced. Copy number polymorphism of the mtDNA pseudogenes was observed among randomly chosen individuals, and even among siblings. A mtDNA pseudogene in the Y-chromosome was observed in a YAC clone carrying only repetitive sequence tag site (STS). PCR screening of human yeast artificial chromosome (YAC) libraries showed that there were at least $5.7 \times 10^{5} \mathrm{bp}$ of the mtDNA pseudo-genes in each haploid nuclear genome. Possible involvement of the mtDNA pseudogenes in the variable part of the human nuclear genome is discussed.
\end{abstract}

Key words: Gene amplification, genome instability, mitochondrial, pseudogene.

\section{INTRODUCTION}

Nuclear pseudogenes of mitochondrial (mt) DNA were initially discovered in the early 80's[1-6]. However, mechanisms for the generation of mtDNA pseudogenes are still not clear and may vary in different cases. Both RNA-[7-8] and DNA mediated[9-11] processes

* Corresponding author: Shanghai Institute of Cell Biology, Chinese Academy of Sciences, 320 Yue Yang Road, Shanghai 200031, China

Tel: 86-21-64378218 Fax: 86-21-64718563 E-mail: hgxgene@sunm.shcnc.ac.cn 
Polymorphism of hum-mtDNA pseudogenes

have been suggested. mtDNA fragments may even act as transposable elements[12, 8] and create new copies of pseudogenes. Evidences showed that some mtDNA pseudogenes had gone through gene amplification in different stages of evolution[13-14]. Our previous studies also showed that some human nuclear mtDNA pseudogenes were multi-copy sequences, probably resulted from (a) very recent gene amplification event(s)[15-16]. In domestic cat, such amplification of mtDNA pseudogenes has lead to tandemly repeated sequences[17-18]. Possible role of mtDNA pseudogenes in carcinogenesis and aging was suggested[8, 19, 20].

The number of mtDNA pseudogenes in the human genome has been estimated to be about a thousand copies, and all regions of mtDNA have nuclear homologies[10, 21]. Here we report 8 nuclear sequence homologous to bp 2477-2593 region of the mt 16S rRNA gene, in addition to those previously reported[15]. Three pseudogenes homologous to the same portion of the $16 \mathrm{~S}$ rRNA gene, designated $\Psi \mathrm{A}, \Psi \mathrm{B}$ and $\Psi \mathrm{C}$, were detected employing a method based on a high-efficiency restriction digestion of cellular DNA and denaturant gradient gel electrophoresis (DGGE). At least two of them have variable copy numbers in different samples. Genome-scale screening for mtDNA pseudogenes in human yeast artificial chromosome (YAC) libraries led to discovery of numerous pseudogenes, one of which was located on a YAC clone carrying only repetitive sequence tag sites (STSs). The overall fraction of mtDNA pseudogene in the nuclear genome was estimated. Our results suggested that the mtDNA pseudogenes could become a variable part of the nuclear genome.

\section{MATERIALS AND METHODS}

\section{Cell, tissue and DNA samples}

The human lymphoblast line TK6 and its cloned population, TK6-KK2, were grown in stirred flasks in RPMI 1640 with 5\% horse serum (JRH Biosciences, Lenexa, KS). Cell lines KB13, 30, 57 and 106 are cybrids of the nuclei from 143B206[22] and the cytoplasms from patients with mitochondrial disease[23]. Cell line 143B12-3A2 is a rho ${ }^{\circ}$ derivative of $143 \mathrm{~B}^{t k}$-[22] derived from a Kirsten murine sacroma virus-transformed human osteosarcoma cell line HOS[24], which was isolated from a 13-year old Caucasian female[25-26]. NA10495A, NA10496A, NA11325, NA11324, NA10846 and NA10847 are DNA samples from National Institute of General Medical Sciences (NIGMS). Human Genetic Mutant Cell Repository (Camden, NJ), isolated from cultured lymphocytes from Pygmy, Chinese and White donors. The human Y chromosome-specific YAC library[27] was purchased from Research Genetics (Huntsville, AL, USA). The CEPH human YAC library[28] was a gift from Dr. Z. Chen, Shanghai Second Medical University, Shanghai, China.

\section{Polymerase-chain reaction $(P C R)$}

PCR was carried out in $10 \mathrm{mM}$ Tris. Cl, pH 8.4; $50 \mathrm{mM} \mathrm{KCl;2.25} \mathrm{mM} \mathrm{MgCl}$; $0.1 \%$ gelatin; 0. $2 \mu \mathrm{M}$ each primer, $150 \mu \mathrm{M}$ dNTPs, $0.1 \mu \mathrm{g} / \mu \mathrm{l} \mathrm{BSA}$, and 5 units Taq DNA polymerase (Perkin Elmer Cetus, Branchburg, NJ) per $100 \mu \mathrm{l}$ reaction. The thermo-cycling of PCR was $94^{\circ} \mathrm{C}, 1 \mathrm{~min} ; 45^{\circ} \mathrm{C}$, $1 \mathrm{~min} ; 72^{\circ} \mathrm{C}, 1 \mathrm{~min}$. Primers used in PCR are Rsma1 (5' AAA AAA AGT AAA AGG AAC TC 3' homologous to bp 2457-2476 of mtDNA[29]); Rsma2A (5' AGG AAC AAG TGA TTA TGC TA 3' homologous to bp 2613-2594 of mtDNA); Rsma3 (5' CTC ACT GTC AAC CCA ACA CA 3' homologous to bp 2415-2434 of mtDNA), Rsma4 (5' TTC ACT GGT TAA AAG TAA GA 3' homologous to bp 2677-2658 of mtDNA); W (5'GAA CTC GGC AAA TGT CGG CC 3' homologous 
Yuan JD et al.

to bp 2471-2490 of mtDNA, mismatches with wild-type mtDNA underlined), and W/GC: 5' CGC CCG CCG CGC CCC GCG CCC GTC CCG CCG CCC CCG CCC G CT CGG CAA AT G TCG GCC' 3' primer W attached to an artificial high-melting temperature GC clamp, which is in italics).

\section{Detection of mtDNA pseudogenes by eliminatingwild-type sequences and $D G G E$}

Cellular DNA was digested with SphI and PvuII. DNA fragments $215+/-40$ bp in length were PAGE purified and recovered by electro-elution using DEAE-cellulose, in order to optimize the restriction efficiency of cellular DNA[30]. Wild-type mtDNA was eliminated by KpnI digestion and indigestible residual DNA was amplified with Taq DNA polymerase using primers $\mathrm{W}$ and RSMA2A. A $5 \mu$ l portion of the reaction was further amplified with primers RSMA2A and 32Plabeled GC/W. Pseudogenes were displayed on a DGGE.

\section{Other technologies}

${ }^{32} \mathrm{P}$-end labeling of oligonucleotides using T4 polynucleotide kinase, digestion of DNA with restriction endonuclease (New England Biolabs, Berely, MA) and DNA sequencing using PCR Product Sequencing Kit (U.S. Biochemical, Cleveland, OH) were performed as per the manufacturer's instructions. PAGE purification of DNA was carried out on a $7 \%$ polyacrylamide gel (acrylamide:bis-acrylamide $=37.5: 1$ ). Gel pieces carrying appropriate DNA fragments were cut from the gel based on an ethidium bromide-stained pBR322/ MspI standard digestion (New England Biolabs, Beverly, MA), so that cellular DNA would not be exposed to ethidium bromide or UV light. DNA in gel pieces was electroeluted onto DEAE-cellulose and recovered by elution with $0.5 \mathrm{ml}$ of $1 \mathrm{M} \mathrm{NaCl}$.DGGE was performed as previously described[31].

\section{RESULTS AND DISCUSSION}

\section{Copy number polymorphism of the nuclear pseudogenes of mtDNA}

Nuclear pseudogenes homologous to bp 2490-2594 region of mtDNA were screened from the total cellular DNA by eliminating the previously sequenced pseudogenes[15, 32] aswellas wild-type mtDNA (see materials and methods). Cellular DNA from two populations of human lymphoblast TK6 and four cybrid lines, KB13, 30, 57 and 106 containing nuclei from 143B206 (a rho ${ }^{\circ}$ derivative of a human osteosarcoma cell line 143Btk-), were examined.

Two DNA sequences, designated $\psi \mathrm{A}$ and $\psi \mathrm{B}$, were detected (Fig 1a). All cybird lines with nuclei from 143B206 had an extra band, $\psi \mathrm{B}$, as compared with TK6. Both $\psi \mathrm{A}$ and $\psi \mathrm{B}$ were observed in the rho ${ }^{\circ}$ cell line 143B12-3A2, a rho ${ }^{\circ}$ cell derivative of 143Btk-[22], confirming that they were nuclear pseudogenes of the $\mathrm{mt}$ 16S rRNA gene, not mutated mtDNA (Fig 1b). The presence of $\Psi \mathrm{A}$ and $\Psi \mathrm{B}$ was also tested in five human blood samples from white donors (data not shown), and in six DNA samples isolated from cultured lymphocytes representing both male and female donors of three races, Pygmy, Chinese and Caucasian (Fig 1b). It was observed that $\psi B$ was detectable only in 9 out of 14 samples tested, while $\psi \mathrm{A}$ was in all of the samples. The intensity ratio of $\psi \mathrm{A}$ verses $\psi \mathrm{B}$ as measured by Phosphorimager (Molecular Dynamics, Sunnyvale, CA) varied from 2.8-0.1 (Fig 1b). It 
Polymorphism of hum-mtDNA pseudogenes

was therefore suggested that the $\psi \mathrm{B}$ is a polymorphic DNA sequence, and its presence was independent on the sex or race of sample donors. However, the ratio between $\psi \mathrm{A}$ and $\psi \mathrm{B}$ remained consistent among the four cybrids of the 143B206, suggesting that the change of the ratio was either infrequent, or took place in meiosis (Fig 1a).

DNA sequencing indicated that $\psi \mathrm{A}$ and $\psi \mathrm{B}$ were $88 \%$ homologous with wild-type mtDNA, and the only difference between the two sequences was that $\psi \mathrm{B}$ carries an extra $\mathrm{A} \rightarrow \mathrm{T}$ mutation at bp 2512 compared to $\psi \mathrm{A}$ (Fig 2 ).

The copy number of $\psi \mathrm{B}$ was further examined in three Chinese pedigrees. The copy number ratio between $\psi \mathrm{A}$ and $\psi \mathrm{B}$ was found to be in general consistent within the pedigrees, suggesting that the copy number variation of $\psi \mathrm{B}$ is probably infrequent even in meiosis. However, another pseudogene, $\psi \mathrm{C}$, was unexpectedly identified and had variable copy numbers even between siblings. The assay was highly reproducible as demonstrated in the quadruplicated assay (Fig 3).

\section{Nuclear pseudogenes of mitochondrial DNA as a variable part of the nuclear genome}

The observation that $\psi \mathrm{B}$ is one base more divergent from wild-type mtDNA than $\Psi \mathrm{A}$ suggested that $\psi \mathrm{B}$ was derived from $\psi \mathrm{A}$ much more recently than the generation of their

a

$$
\text { 苨 }
$$

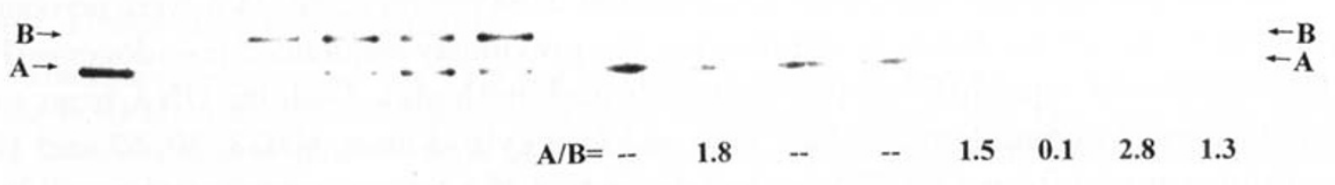

Fig 1.

Pseudogenes $\psi \mathrm{A}$ and $\psi \mathrm{B}$ displayed on a DGGE. Cellular DNA from cultured cells (panel a) and from NIGNS DNA samples (panel b) were tested as described in ATERIALS AND METHODS Pseudogenes $\psi \mathrm{CA}$ and $\psi \mathrm{CB}$ were labeled with arrows. Sample names were indicated on top of each lane. The intensity ratios between bands $\mathrm{A}$ and $\mathrm{B}$ of the samples shown in panel b were measured by Phosphorimager $^{T M}$ analysis (Molecular Dynamics, Sunnyvale, CA). Pygmy male and female, Chinese male and female, and White male and female were NA10495A, NA10496A, NA11325, NA11324, NA10846 and NA10847 from National Institute of General Medical Sciences (NIGMS), Human Genetic Mutant Cell Repository (Camden, NJ).

$\triangleright$ Fig 2.

DNA sequences of wild-type mtDNA and mtDNA pseudogenes. The GeneBank accession numbers were labeled next to the names of the pseudogene sequences. Numbers indicated the base position in the Cambridge sequence [Anderson et al. 1981]. The recognition site for pnKpnI was in italic. Sequences to which primers Rsma3, Rsma1, W, Rsma2A and Rsma4 combine were underlined. 
2415

CTCACT GTCAACCCAA CACAGGCATG CTCATAAGGA AAGGTTAAAA

Rsma3

- T- - - - - - - - - T-A----

$\Psi \mathbf{Y}$

2461

AAAGTAAAAG GAACTCGGCA AATCTTACCC CGCCTGTTTA CCAAAAACAT Rsma1

$\mathrm{W}$

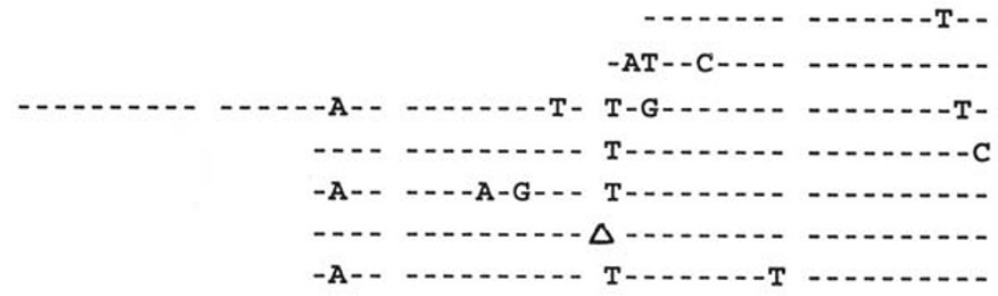

$\Psi_{\mathrm{A} / \mathrm{L} 27603}$

$\Psi_{B / L 27602}$

$\Psi_{C} / \mathrm{AF} 055272$

$\Psi \mathrm{Y} / \mathrm{AF} 055271$

$963 B 7 / A F 056112$

$964 \mathrm{~F} 2 / \mathrm{AF} 056113$

699 H8/AF0 56114

$965 \mathrm{~F} 2 / \mathrm{AF} 056115$

2511

CACCTCTAGC ATCACCAGTA TTAGAgGCAC CGCCTGCCCA GTGACACATG

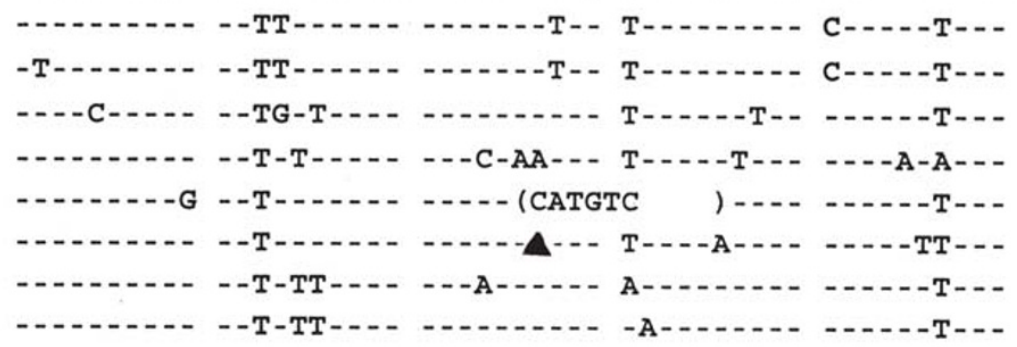
$\Psi_{\mathrm{A} / \mathrm{L} 27603}$
$\Psi_{B / L 27602}$
$\Psi \mathrm{C} / \mathrm{AF} 055272$
$\Psi \mathrm{Y} / \mathrm{AF} 055271$
963B7 / AF056112
$964 \mathrm{~F} 2 / \mathrm{AF} 056113$
699 H8/AF056114
965F2/AF056115

2561

TTTAACGGCC GCGGTACCCT AACCGTGCAA AGGTAGCATA ATCACTTGTT

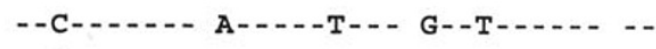

Rsma2a

$\Psi_{\mathrm{A} / \mathrm{L} 27603}$

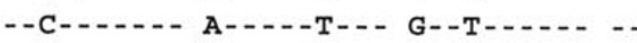

$\Psi_{\mathrm{B} / \mathrm{L} 27602}$

$---G--T T----T--$ G-A-T-D- -

$\Psi_{\mathrm{C} / \mathrm{AF} 055272}$

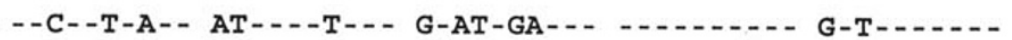

$\Psi \mathrm{Y} / \mathrm{AF} 055271$

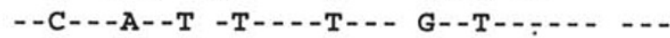

963B7/AF056112

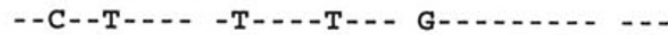

964 F $2 / A F 056113$

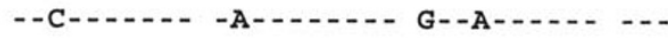

699 H8/AF056114

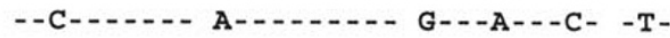

$965 \mathrm{~F} 2 / \mathrm{AF} 056115$

2611

CCTTAAATAG GGACCTGTAT GAATGGCTCC ACGAGGGTTC AGCTGTCTCT

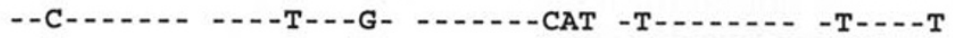

$\Psi \mathrm{Y} / \mathrm{AF} 055271$

2661

TACTTTTAAC CAGTGAAATT GACCTGCCCG

Rsma4

Fig 2 . 


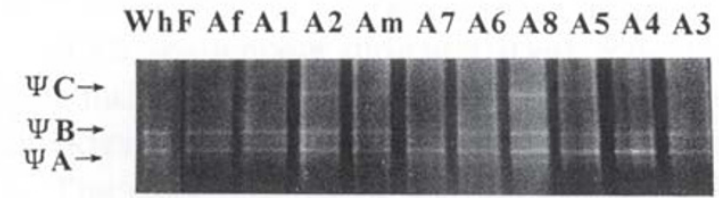

a

C5 C4 C2 Cm Cf C6 C1 C3 WhF

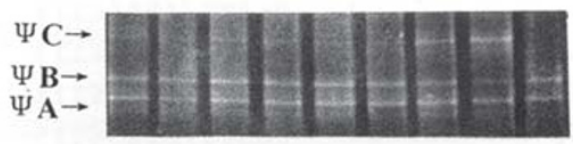

C

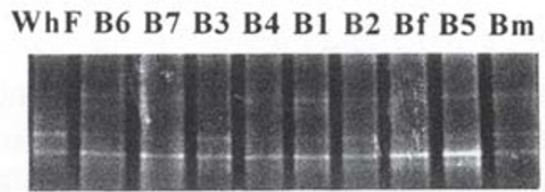

b

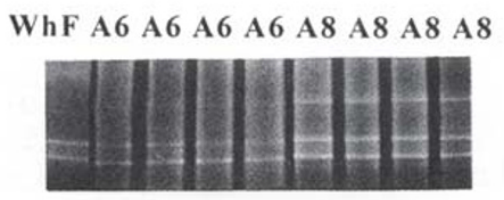

$\mathbf{r}$

Fig 3.

Distribution of pseudogenes $\psi \mathrm{A}, \psi \mathrm{B}$ and $\psi \mathrm{C}$ in pedigrees. Samples were treated the same way as described in Fig 1 except that the DGGE gels were ethidium bromide-stained. Panels a, $\mathrm{b}$ and $\mathrm{c}$ were the DGGE display of pseudogenes from pedigrees A, B, and C. f, father; m, mother. The numbered samples in each pedigree represented the children from the parents $f$ and $\mathrm{m}$. WhF was the white female sample in Fig 3. Panel $r$ was a quadruplicated study indicating that the variable intensity ratio between pseudogenes $\psi \mathrm{A}, \psi \mathrm{B}$ and $\psi \mathrm{C}$ were highly reproducible and was not an experimental artifact. The ratios measured by phosphorimager ${ }^{T M}$ analysis were WhF (1/1.2/0); A6(6/6/1); (6/6/1); $(6 / 6 / 1.2)(6 / 6 / 1) ; A 8(1 / 1 / 0.9) ;(1 / 1 / 1) ;(1 / 1 / 0.9) ;(1 / 1.1 / 0.9)$

common ancestor sequence. This is consistent with observations from other mtDNA pseudogenes[1, 13-16]. During the in vitro enzymatic amplification, $\psi \mathrm{A}, \psi \mathrm{B}$ and $\psi \mathrm{C}$ served as quantitative internal standards of each other, which should accurately reflect their relative gene dosage even at the plateau phase of PCR[32-34]. The variable intensity ratio between $\psi \mathrm{A}, \psi \mathrm{B}$ and $\psi \mathrm{C}$ thus implied that all three pseudogenes might be multi-copy sequences, and that the copy number of at least two of the pseudogenes differed among the samples. The fact that $\psi \mathrm{A}$ presents in all samples lead to the hypothesis that $\Psi \mathrm{B}$ and $\psi \mathrm{C}$ were the ones with variable copy numbers, although the possibility that all three pseudogenes had copy number polymorphisms could not be ruled out. We thus suggest that mtDNA pseudogenes might have been involved in the variable part of the human genome.

\section{PCR screening of YAC libraries for mtDNA pseudogenes}

We were very interested in the mechanism(s) by which the copy number of mtDNA pseudogenes changed. Cloning of those multi-copy pseudogenes would help to understand the related gene-amplification mechanisms. However, direct cloning a particular pseudogene 
via any hybridization-based technology was proven unsuccessful (data not shown). Major causes of the failure included the interference from the wild-type mtDNA fragments cloned in most genomic libraries, the presence of a large number of pseudogenes homologous to the same part of the mtDNA and the instability of the cloned multicopy pseudogenes. An alternative approach we took was to screen human YAC libraries for multi-copy pseudogenes by PCR[15], expecting some YAC clones might have maintained some repetitive sequences.

From a Y chromosome-specific YAC library[27], a YAC clone \#143 was found to carry a new mtDNA pseudogene, designated $P$ si Y. PCR amplification using primers Rsma3 and Rsma4 give rise to a longer sequence as shown in Fig 2. It was interesting that the YAC clone \#143 could not be located on a particular position of the Y chromosome because it contained only repetitive STSs. The observation was consistent with the hypothesis that the mtDNA pseudogene was involved in the variable part of the nuclear genome.

Similar screening of 105 YAC pools, each containing 96 YAC clones which carried 800$1200 \mathrm{~kb}$ genomic DNA each, from the CEPH human YAC library using primers Rsma1 and Rsma2a yielded a large number of pseudogenes. Those pseudogenes, after being further amplified with Rsma2a and a nested primer W/GC, were displayed on a denaturant gradient gel electrophoresis (DGGE). On average, one pseudogene that has a melting temperature different from that of the wild-type mtDNA could be amplified from each 100 YAC clones, which was approximately 108 bp of human genomic DNA (data not shown), or 1/30 of a haploid human genome. Four of them from the YAC clones 963B7, 964F2, $699 \mathrm{H} 8$ and $965 \mathrm{~F} 2$ were sequenced (Fig 2). However, none of the known multi-copy pseudogenes was detected from the YAC libraries, probably due to the instability of the YAC clones containing the repetitive pseudogene sequences.

The PCR product amplified by primers Rsma1-Rsma2a is $157 \mathrm{bp}$ in length, and the average length of the published uninterrupted mammalian mtDNA pseudo-genes was 1228bp[13, 21, 35-37] (including those we identified in GenBank-deposited human genomic sequences, GenBank accession number U66061, bp46495-48989; AF029308, bp5019952715; and AC004035, bp5778-7569, bp7870-9657, bp9990-10604 and bp10924-11009). If that is typical in all mtDNA pseudogenes, a mtDNA pseudo-gene should have (1228-157)/ $16569=6.46 \%$ of chance to be amplified by a given primer pair that generates a $157 \mathrm{bp}$ PCR product. Assuming that most part of the mtDNA has the similar chance to be integrated into the nuclear genome, each pseudogene amplified by the primers Rsma1-Rsma2a should represent $1228 / 6.46 \%=1.9 \times 10^{4} \mathrm{bp}$ of mtDNA-like sequence in the chromosomes. The amount of the mtDNA-like sequence in the human haploid genome can thus be estimated to be at least $1.9 \times 10^{4} \times 30=5.7$ times $10^{5}$, or 35 equivalents of the complete $\mathrm{mt}$ genome. Considering the presence of the multi-copy pseudogenes and the pseudogenes that might be identical to the wild-type sequence[16], the real fraction of the mtDNA pseudogenes in the human nuclear genome should be even greater.

We observed mtDNA pseudogenes with variable copy numbers, and estimated the fraction of mtDNA pseudogenes in the nuclear genome. It was known that microsatellite 
Polymorphism of hum-mtDNA pseudogenes

DNA changed copy numbers via intra-allelic duplications/deletions and inter-allelic recombination/conversions depending on flanking sequences[38-40]. It would be interesting to find out whether the mtDNA pseudogenes changed copy numbers as minisatellite DNA did. Understanding the flanking sequence of the multi-copy pseudogenes, especially those with variable copy numbers such as $\Psi \mathrm{B}$ and $\Psi \mathrm{C}$, would provide more information on how mtDNA pseudogenes had been generated during evolution, and how they might affect the genetic stability of the human nuclear genome.

\section{ACKNOWLEDGEMEN}

We thank Dr. Michael KING for providing us with 143B12-3A2, KB13, 30, 57 and 106 cell strains, and Dr. Zhu CHEN for the CEPH YAC clones. This research was supported by the Grant for Major International Collaborative Research Project from the Chinese Academy of Sciences.

\section{REFERENCES}

[1] Boogaart Pvd, Samallo J, Agsteribbe E. Similar genes for a mitochondrial ATPase subunit in the nuclear and mitochondrial genomes of Neurospora crassa. Nature 1982; 298:187-9.

[2] Farrelly F, Butow RA. Rearranged mitochondrial gene in the yeast nuclear genome. Nature 1983; 301: 296-301.

[3] Gellissen G, Bradfield JY, White BN, Wyatt GR. Mitochondrial DNA sequences in the nuclear genome of a locust. Nature 1983; 301:631-4.

[4] Hadler HI, Dimitrijevic B, Mahalingam R. Mitochondrial DNA and nuclear DNA from normal rat liver have a common sequence. Proc Natl Acad Sci USA 1983; 80:6495-9.

[5] Tsuzuki T, Nomiyama H, Setoyama C, Maeda S, Shimada K. Presence of mitochondrial-DNA-like sequences in the human nuclear DNA. Gene 1983; 25:223-9.

[6] Wright RM, Cummings DJ. Integration of mitochondrial gene sequences wtihin the nuclear genome during senescence in a fungus. Nature 1983; 302:86-8.

[7] Gellissen G, Michaelis G. Gene Transfer. Mitochondria to nucleus. Ann N Y Acad Sci 1987; 503:391401.

[8] Shay JW, Werbin H. New evidence for the insertion of mitochondrial DNA into the human genome: significance for cancer and aging. Mutat Res 1992; 275:227-35.

[9] Jacobs HT, Grimes B. Complete nucleotide sequences of the nuclear pseudogenes for cytochrome oxidase subunit I and the large mitochondrial ribosomal RNA in the sea urchin Strongylocentrotus purpuratus. J Mol Biol 1986; 187:509-27.

[10] Kamimura N, Ishii S, Liandong M, Shay JW. Three separate mitochondrial DNA sequences are contiguous in human genomic DNA. J Mol Biol 1989; 210:703-7.

[11] Thorsness PE, Fox TD. Escape of DNA from mitochondria to the nucleus in Saccharomyces cerevisiae. Nature 1990; 346:376-9.

[12] Kemble RJ, Mans RJ, Gabay-Laughnan S, Laughnan JR. Sequences homologous to episomal mitochondrial DNAs in the maize nuclear genome. Nature 1983; 304:744-7.

[13] Zullo S, Sieu LC, Slightom JL, Hadler HI, Eisenstad JM. Mitochondrial D-loop sequences are integrated in the rat nuclear genome. J Mol Biol 1991; 221:1223-35.

[14] Smith MF, Thomas WK, Patton JL. Mitochondrial DNA-like sequence in the nuclear genome of an akodontine rodent. Mole Biol Evol 1992; 9:204-15.

[15] Hu G, Thilly WG. Evolutionary trail of the mitochondrial genome as based on human 16S rDNA 
pseudpogenes. Gene 1994; 147:197-204.

[16] Hu G, Thilly WG. Multi-copy nuclear pseudogenes of mitochondrial DNA reveal recent acute genetic changes in the human genome. Curr Genet 1995; 28:410-4.

[17] Lopez JV, Cevario S, O'Brien SJ. Complete nucleotide sequences of the domestic cat (Feliscatus) mitochondrial genome and a transposed mtDNA tandem repeat (Numt) in the nuclear genome. Genomics 1996; 33:229-46.

[18] Lopez JV, Yuhki N, Masuda R, Modi W, O'Brien SJ. Numt, a recent transfer and tandem amplification of mitochondrial DNA to the nuclear genome of the domestic cat. J Mol Evol 1994; 39:174-90.

[19] Reid RA. Can migratory mitochondrial DNA activate oncogenes? Trends Biochem Sci 1983; 8:190-1.

[20] Richter C. Do mitochondrial-DNA fragments promote cancer and aging? FEBS Lett 1988; 241:1-5.

[21] Fukuda M, Wakasugi S, Tsuzuki T, Nomiyama H, Shimada K, Miyata T. Mitochondrial DNA-like sequences in human nuclear genome: Characterization and implications in the evolution of mitochondrial DNA. J Mol Biol 1985; 186:257-66.

[22] King MP, Attardi G. Human cells lacking mtDNA: Repopulation with exogenous mitochondria by complementation. Science 1989; 246:500-3.

[23] Masucci JP, Davidson M, Koga Y, Schon EA, King MP. In vitro analysis of mutants causing myoclonus epilepsy with ragged-red fibers in the mitochondrial tRNALys gene: two genotypes produce similar phenotypes. Mol Cell Biol 1995; 15:2872-81.

[24] Croce CM, Barrick J, Linnenbach A, Koprowski H. Expression of malignancy in hybrids between normal and malignant cells. J Cell Physiol 1979; 99:279-85.

[25] Rhim JS, Cho HY,Huebner RJ. Non-producer human cells induced by murine sarcoma virus. Int J Cancer 1975a; 15:23-9.

[26] Rhim JS, Cho HY, Vernon ML, Arnstein P, Huebner RJ, Gilden RV. Characterization of non-producer human cells induced by Kirsten sarcoma virus. Int J Cancer 1975b; 16:840-9.

[27] Foote S, Vollrath D, Hilton A,Page DC. The human Y chromosome: Overlapping DNA clones spanning the euchromatic region. Science 1992; 258:60-6.

[28] Albertsen H, Abderrahim H, Cann H, Dausset J, Le-Paslier D, Cohen D. Construction and characterization of a yeast artificial chromosome library containing seven haploid human genome equivalents. Proc Nat Acad Sci USA 1990; 87:4256-60.

[29] Anderson S, Bankier AT, Barrell BG et al. Sequence and organization of the human mitochondrial genome. Nature 1981; 290:457-65.

[30] Khrapko K, Andre P, Cha R, Hu G, Thilly WG. Mutational Spectrometry: Means and ends. Prog Nucl Acad Res Mol Biol 1994; 49:285-312.

[31] Keohavong P, Thilly WG. Fidelity of DNA polymerases in DNA amplification. Proc Natl Acad Sci USA 1989; 86:9253-7.

[32] Celi FS, Cohen MM, Antonarakis SE, Wertheimer E, Roth J, Shuldiner AR Determination of gene dosage by a quantitative adaptation of the polymerase chain reaction (PCR): rapid detection of deletions and duplications of gene sequences. Genomics 1994; 21:304-10.

[33] Cottrez F, Auriault C, Capron A, Groux H. Quantitative PCR: validation of the use of a multispecific internal control. Nucl Acid Res 1994; 22:2712-3.

[34] Morrison C, Gannon F. The impact of the PCR plateau phase on quantitative PCR. Biochim Biophy Acta $1994 ;$ 1219:493-8.

[35] Nomiyama H, Fukuda M, Wkasugi S, Tsuzuki T, Shimada K. Molecular structures of mitochodnrialDNA-like sequences in human nuclear DNA. Nucl Acid Res 1985; 13:1649-58.

[36] Zischler H, Geisert H, Haeseler Av, Paabo A. A nuclear 'fossil' of the mitochondrial D-loop and the origin of modern humans. Nature 1995; 378:489-92.

[37] Rowen L, F KB, Hood L. The complete 685-kilobase DNA sequence of the human bate T cell receptor locus. Science 1996; 272:1755-62.

[38] Jeffreys AJ, Wilson V, Thein SL. Hypervariable 'minisatellite' regions in human DNA. Nature 1985; 


\section{Polymorphism of hum-mtDNA pseudogenes}

314:67-73.

[39] Jeffreys AJ, Tamaki K, MacLeod A, Monckton DG, Neil DL, Armour JA. Complex gene conversion events in germline mutation at human minisatellites. Nat Genet 1994; 6:136-45.

[40] Monckton DG, Neumann R, Guram T et al. Minisatellite mutation rate variation associated with a flanking DNA sequence polymorphism. Nat Genet 1994; 8:162-70.

Received Nov-11-1999. Revised Nov-18-1999. Accepted Nov-26-1999. 